\title{
FORMULATION AND EVALUATION OF NIOSOMAL SUSPENSION OF CEFIXIME
}

\section{BHAMA SANTHOSH KUMAR, RAKHI KRISHNA, LAKSHMI PS, DEEPA T VASUDEV, SREEJA C NAIR*}

Department of Pharmaceutics, Amrita School of Pharmacy, Amrita Vishwa Vidyapeetham, Amrita University, AIMS Health Sciences Campus, Kochi, Kerala, India. Email: sreejacnair@aims.amrita.edu

Received: 19 January 2016, Revised and Accepted: 14 February 2017

ABSTRACT

Objectives: The present study was aimed to overcome the problems associated with the drug such as bioavailability, to reduce the dosage regimen, half-life, and to determine the appropriateness of niosomal formulation as a drug carrier.

Methods: The niosomal suspension was prepared by thin film technique, by varying ratios of span 60 and cholesterol and varying the concentration of span 60 . The prepared four formulations were evaluated for various parameters.

Results: The optimized formulation had a vesicular size of 250-400 nm. Varying the concentration of span 60, the entrapment efficiency demonstrated that it had a considerable task. The highest entrapment efficiency was $95.3 \%$. The kinetics study confirmed that the liberation of drug from the niosomal suspension is in a restricted manner. The statistical optimization showed that NS2 is the optimized formulation. The gastrointestinal enzymes showed no significant change in the release of drug from the formulation. The zone of inhibition showed that optimized formulation has a better activity than the marketed formulation. The MIC was found to be $0.05 \mathrm{mg}$, hence can be used as an efficient carrier for delivery of cefixime.

Conclusion: The present study concludes that the prepared niosomal suspension is a convenient and efficiency carrier for the delivery of antibacterial drug. Besides this, it provided controlled delivery of drug.

Keywords: Niosomes, Cholesterol, Thin film technique, Vesicular size, Controlled drug delivery.

(C) 2017 The Authors. Published by Innovare Academic Sciences Pvt Ltd. This is an open access article under the CC BY license (http://creativecommons. org/licenses/by/4. 0/) DOI: http://dx.doi.org/10.22159/ajpcr.2017.v10i5.17189

\section{INTRODUCTION}

Noisomes are non-ionic surfactant of the alkyl or dialkyl polyglycerol ether class and cholesterol with subsequent hydration in aqueous media. These are lamellar structures that are microscopic in size. Structurally niosomes are bilayered in nature. On the basis of preparation methods used, niosomes may be unilayer or multilayer [1,2]. Depending on various factors such as stability and cost, the niosomes are considered ideal when compared with liposomes. For various routes such as topical, ophthalmic, and parenteral niosomes prove to be a potential and possible drug delivery mechanism [3]. It was prepared by the method of ether injection method, handshaking method (thin film hydration technique), sonication, microfluidization, multiple membrane extrusion method, reverse phase evaporation technique, transmembrane $\mathrm{pH}$ gradient (inside acidic) drug uptake process (remote loading), and the "Bubble" method [4-8]

Various factors affecting niosomal formulations are type of surfactant, drug and amount, charge and cholesterol content, osmotic stress due to resistance, and constitution of membrane [6,9-12].

The main aim of this project is to study about niosomal suspension which is emerging as a potential drug carrier, as a new drug delivery for the antibiotic drug cefixime. Therefore, the objective behind the study is to prolong the action by incorporating the drug into the vesicular carriers.

\section{MATERIALS AND METHODS}

\section{Methodology}

\section{Pre-formulation studies}

Pre-formulation investigations are to provide information on physicochemical and biopharmaceutical study properties of drug molecule, non-drug substance, and materials used for packaging as well as compressibility.

\section{Identification of the drug}

\section{Melting point}

The purity of the sample is indicated by the melting point so obtained. The melting point values can get lowered because of the presence of unwanted substances

\section{$\lambda_{\max }$ of the drug}

Using phosphate buffer, the absorption maximum of cefixime was obtained. A range of solutions $(2-10 \mu \mathrm{g} / \mathrm{ml})$ was scanned using UV spectrophotometer.

\section{Solubility}

To obtain a desirable concentration of the drug in the blood, solubility plays a major role [13]. Varying solvents were taken including $0.1 \mathrm{~N}$ HCL, methanol, water, ethanol, and certain buffers such as phosphate buffer (7.4) to determine the solubility.

\section{Partition coefficient of drug}

Partition coefficient of cefixime in n-octanol-water was obtained.

Physical drug excipient compatibility

At $75 \%$ relative humidity and $40^{\circ} \mathrm{C}$, the formulations were kept sealed for 30 days. Then, it was compared with the initial state for any occurrence of interaction.

Fourier transform infrared (FTIR) studies

With the help of infrared absorption spectral analysis (FTIR), the cefixime compressibility with individualized non-drug component and physical combination of the major formulation was produced. Spectral analysis using IR helped to identify any modification in the characters of the formulation when it was mixed with the non-drug substance. 


\section{Formulation of niosomal suspension}

Method

Niosomal suspension was prepared by thin film hydration method (Table I). The cholesterol and the surfactant were mixed and added in about $20 \mathrm{ml}$ of chloroform into an evaporating flask of rotary evaporator and dipped in water bath at $300^{\circ} \mathrm{C}$. It was then evaporated at very low pressure until it formed a dry thin film on the flask, which was then dissolved in $20 \mathrm{ml}$ of phosphate buffer, and kept on a water bath at $450^{\circ} \mathrm{C}$ and $120 \mathrm{rpm}$ rotation to hydrate the layers, later sonicated to obtain niosomal suspension. The prepared niosomal suspension was then stored in a tight container in refrigerator for further characterization [10].

\section{Characterization of niosomal suspension}

Vesicular size evaluation

Microscopic evaluation

A little amount of niosomal suspension was dissolved with 10-12 ml of phosphate buffer ( $\mathrm{pH}$ 7.2). The distribution of niosomal suspension was shaken in regular intervals of time. When an drop of dispersion was microscopically observed, rounded vesicular constituents were seen with uniform small size at $\times 100$.

\section{Size and size distribution}

Dynamic light scattering method was used to evaluate vesicular size distribution. The niosomal suspension was diluted with 10-12 ml distilled water with physical shaken. The temperature was fixed at $20^{\circ} \mathrm{C}$, refractive index at 1.333 viscosity, and 0.01 poise.

\section{Scanning electron microscopy}

Niosomal suspension was diluted with distilled water and was attached with the help of a glued double-faced carbon tape on an aluminum stub. With the help of a vacuum evaporator, the vesicular components covered with gold and were examined.

\section{Entrapment efficiency}

The niosomal suspension was sonicated after loading the drug along with the phosphate buffer and was centrifuged and separated. Assay was done at $288 \mathrm{~nm}$ to quantify cefixime.

Zeta potential determination

The ideal formulation's zeta potential was obtained by Zetasizer.

\section{In vitro drug release}

It carried out for the four niosomal suspension formulations.

\section{Activation of cellophane membrane}

The cellophane membrane was activated first by dipping in hot water for $1 \mathrm{hr}$, and then membrane was immersed in ethanol for 30 minutes. The ethanol-immersed membrane was then transferred to acetate buffer of pH 4 and kept for overnight.

\section{In vitro drug release studies in gastrointestinal $\mathrm{pH}$}

Three milliliters of niosomal suspension containing known amount of drug was taken in a cellophane membrane tied to one of the ends of an open-ended cylinder. The entire apparatus was immersed in $30 \mathrm{ml}$ $0.1 \mathrm{~N} \mathrm{HCL} \mathrm{pH} 1.2$ and stirred at $370^{\circ} \mathrm{C}$. Samples of definite amount were withdrawn at specific intervals of time $(5,10,15,30,45,60,75,90$, 105 , and 120 minutes) and were analyzed by UV spectrophotometry at $288 \mathrm{~nm}[11,12]$

In vitro drug release studies in phosphate buffer $\mathbf{p H ~} 7.2$

Using spectrophotometry, the percentage of drug released was obtained.

\section{Statistical design of experiment}

The software was used to perform the procedure for statistical optimization. The statistical optimization procedure was performed with the help of optimization software such as Statgraphics Centurion 16
ANOVA study by one-way method

When the $\mathrm{F}$ value is higher than critical $\mathrm{F}$ value, the null hypothesis is rejected.

Study on the effect of gastrointestinal enzymes on the release of drug

The effect of the enzymes such as trypsin and pepsin was studied on the release of optimized formulation. $2 \mathrm{ml}$ of niosomal suspension containing known amount of drug was taken in a cellophane membrane tied to one of the ends of an open-ended cylinder. The entire apparatus was immersed in $30 \mathrm{ml} 0.1 \mathrm{~N}$ HCL pH 1.2 containing $0.25 \%$ pepsin and stirred at $370^{\circ} \mathrm{C}$. Samples of definite amount were withdrawn at specific intervals of time $(5,10,15,30,45,60,75,90,105$, and 120 minutes $)$ and were analyzed by UV spectrophotometry at $288 \mathrm{~nm}[13,14]$.

\section{In vitro antibacterial activity}

The in vitro antibacterial activity was performed using agar diffusion assay method. The microorganism employed was Escherichia coli and Klebsiella. The activity was performed on optimized formulation, marketed formulation (cefixime - oral suspension), and plain niosomal suspension.

\section{Media and Chemicals}

Media and chemicals were distilled water, 05\% sodium chloride, 1.5\% agar, $0.5 \%$ peptone, $0.3 \%$ beef extract/yeast extract, and $\mathrm{pH}$ adjusted to neutral (6.8) at $25^{\circ} \mathrm{C}$.

\section{Additional materials required}

Additional materials required were standard microbiological supplies and apparatus such as biochemical reagents, incubators, incinerator/ autoclave, microscope, staining suppliers, slides, saline blank, and loops.

Comparison study of antibacterial activity of optimized formulation with marketed formulation

The antibacterial activity of the ideal formulation was studied against commercially available formulation of the drug. $0.1-0.2 \mathrm{ml}$ of the test microorganism was inoculated into the freshly prepared sterile nutrient agar plates. Wells were arranged, and a positive control was also added to another well. The plates were incubated for 18-48 hrs at room temperature. After incubation, they were examined for the presence or absence of the growth $[15,16]$.

Determination of minimum inhibitory concentration (MIC)

The bacterial activity was inhibited by the increase in the concentration of the sample. The MIC was taken at the lowest concentration that prevented the growth of the microorganisms [17].

\section{Stability studies}

The ideal formulation of NS2 was preserved for 45 days at two different temperatures $30 \pm 2^{\circ} \mathrm{C}$ and $4 \pm 2^{\circ} \mathrm{C}$ to evaluate chemical and physical stabilities. For $7,15,30$, and 45 days, the formulation was evaluated for entrapment efficiency [18-20].

\section{RESULT AND DISCUSSION}

\section{Melting point of the drug}

In accordance with the monograph, the melting point of the drug was found $\left(218-225^{\circ} \mathrm{C}\right)$.

\section{$\lambda_{\max }$ of the drug}

The $\lambda_{\max }$ of the drug was found to be $288 \mathrm{~nm}$, and it was in agreement with the official standard as in Fig. 1.

The above parameters such as melting point, $\lambda_{\text {ma, }}$ and FTIR showed that it was in accordance with the reference and hence pure sample of cefixime I.P.

\section{Solubility of the drug}

The pure drug solubility was compared with the standard sample, and it reveals that the pure drug is highly soluble in methanol (95\%) and practically insoluble in water. 
Partition coefficient of the drug

The partition coefficient of the drug was found to be 3.2. This indicates that cefixime is practically insoluble in water and having high lipid solubility, so this can be incorporated into niosomes for the efficient transport.

Physical drug excipient compatibility

There were no incompatibilities or interaction between drug and excipients.

\section{FTIR spectroscopy}

From the spectra, it is evident that all the peaks in the FTIR of the drug are shown (Fig. 2) in the FTIR peak of the formulation. This finding confirms that cefixime does not interact with any of the ingredients of the niosomal suspension.

\section{Analytical method}

Calibration curve (Fig. 2) in phosphate buffer $\mathrm{pH} 7.2$ shows the absorption reading of standard drug solution containing $2-10 \mu \mathrm{g} / \mathrm{ml}$ of drug in phosphate buffer (7.2) at a wavelength of $288 \mathrm{~nm}$.
On the basis of this standard curve (Fig. 3), the calculations of drug contents and in vitro drug release are studied.

\section{Characterization of niosomal suspension}

- Vesicular size evaluation

- Microscopic evaluation

Dissolved niosomes from niosomal suspension were observed microscopically and found to be having similarity and uniformity in vesicle size.

\section{Size and size distribution}

When evaluated for vesicular size and size distribution, it showed that ionic concentration rise can increase the mean vesicular size which ranged between 215 and $400 \mathrm{~nm}$. The niosomal suspensions formed by hydration were of uniform size as suggested by the low polydispersity index as shown in Fig. 4.

\section{Scanning electron microscopy}

Fig. 5 shows that the optimized formulation has a particle size between to be 250 and $400 \mathrm{~nm}$.

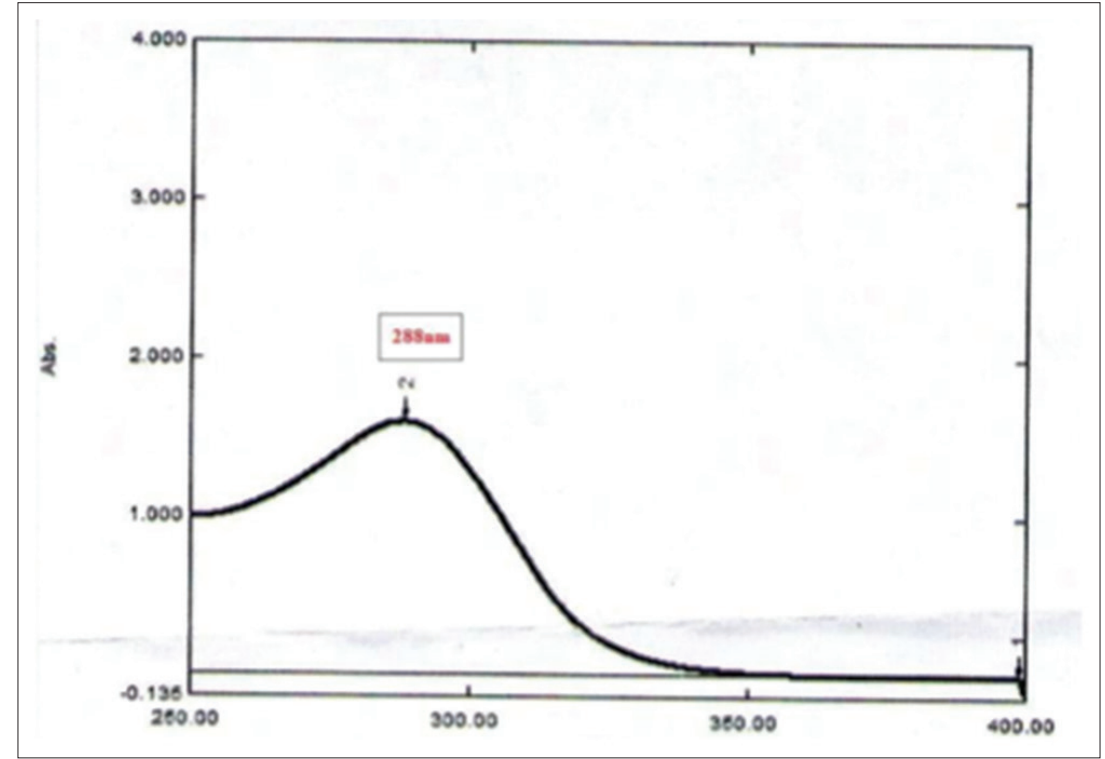

Fig. 1: Absorption maxima of cefixime in phosphate buffer in phosphate buffer $(\mathrm{pH}=7.2)$

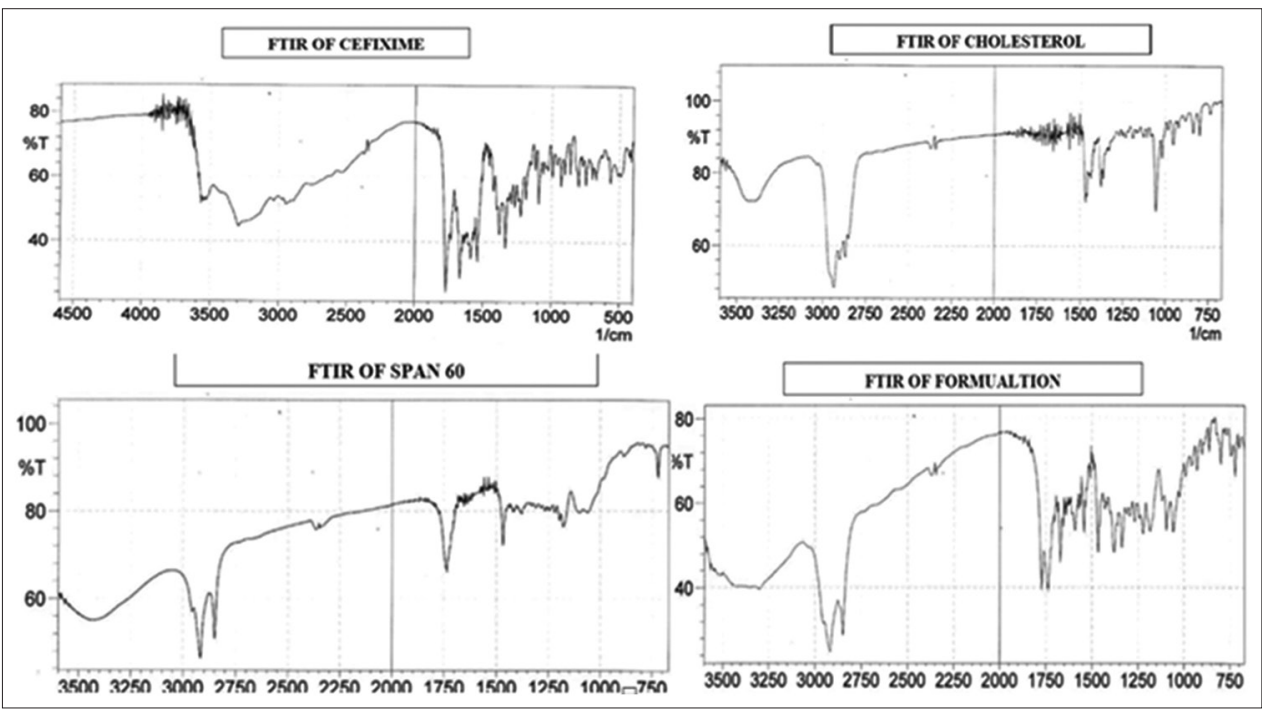

Fig. 2: Fourier transform infrared of cefixime, cholesterol, span 60, formulation 


\section{Entrapment efficiency}

Entrapment efficiency of these formulations was found considerable at $\mathrm{p}<0.05$ (Fig. 6). Depicts the entrapment efficiency of the different formulations. NS2 has the highest entrapment efficiency.

\section{Zeta potential determination}

The zeta potential determination confirmed that the ideal formulation (NS2) illustrates that the sample is stable suspension.

\section{In vitro release studies}

Both in vivo and delivery system functioning inside the body can be predicted by the release profile of a drug. All the four formulations underwent in vitro release studies in stomach $\mathrm{pH}$ and intestinal $\mathrm{pH}$. The release data represented in the graph in Figs. 7-9.

The in vitro release pattern of the four formulations in the Stomach $\mathrm{pH}$ was studied, and it was concluded that there is no significant release of drug in the stomach $\mathrm{pH}$ as the release is only $0.4 \%$. The release profiles suggest that the release of drug occurs slowly and later immediate release. Therefore, the release first occurs slowly and then immediately due to penetration enhancement of nonionic surfactant.

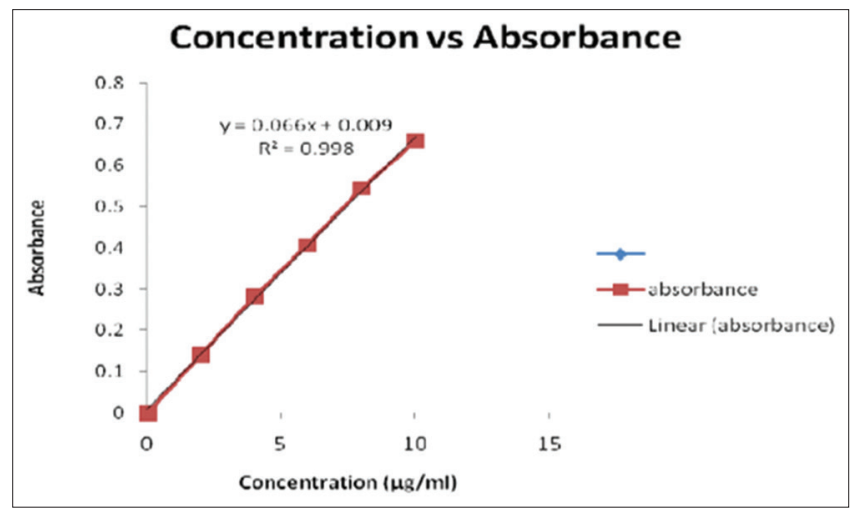

Fig. 3: Calibration in phosphate buffer pH 7.2
Kinetic modeling of in vitro drug release

The in vitro drug liberation of NS2 was fitted into various kinetic equations, and the linearity of each plot was determined by the closeness of regression coefficient to unity. The kinetic study of the release data showed that NS2 showed Higuchi model as the best fit model. The linearity of Higuchi plots indicates that as the distance of the diffusion increases the release of drug will be at a slower pace. This concludes that the drug is being released from the inner side of niosomal suspension in a restricted method. Kinetic study of the drug release profile optimum formulations NS2 is shown, and the graphical representations are shown in Fig. 9.

Optimization of formulation by one-way ANOVA method

Analysis of variance was used to optimize the formulation; it was carried out at $95 \%$ confidence interval. The report is represented in Figa. 10 and 11.

\section{ANOVA analysis report}

The ANOVA of entrapment efficiency indicates that both surfactant and cholesterol are significant terms as the $\mathrm{F}$ values are above the critical $\mathrm{F}$ values and thus making the $\mathrm{p}<0.05$ (threshold level).

There is a statistically significant difference between the means of the two variables at the $95.0 \%$ confidence level, represented in Fig. 12

Study on the effect of gastrointestinal enzymes on the release of drug

In vitro antibacterial activity

a. Comparison study of antibacterial activity of the optimized formulation and marketed formulation by zone of inhibition.

The optimized formulation NS2 was found to have better activity in both E. coli and Klebsiella than the marketed formulation of the drug (Table 2).

b. Determination of MIC of cefixime loaded niosomal suspension (Fig. 13).

The MIC of the optimized formulation was obtained.

Furthermore, the value is tabulated in Table 3.
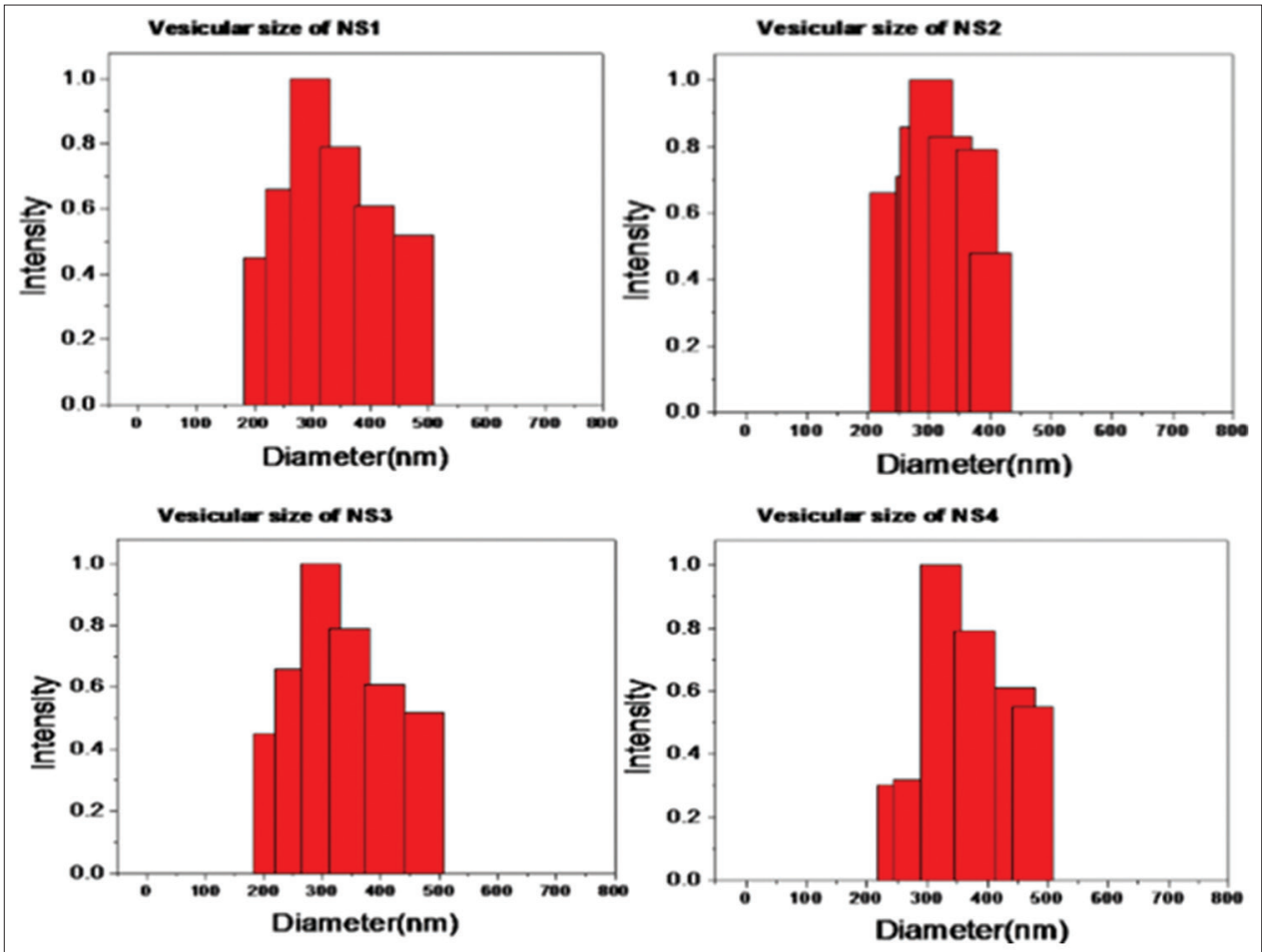

Fig. 4: Vesicular size of niosomal suspension of NS1, NS2, NS3, and NS4 
Stability studies

The optimized formulation at two temperatures, i.e. $30 \pm 2^{\circ} \mathrm{C}$ and $4 \pm 2{ }^{\circ} \mathrm{C}$ were done for 45 days; it does not show any variation when studied against the newly prepared formulation. There is no major variation

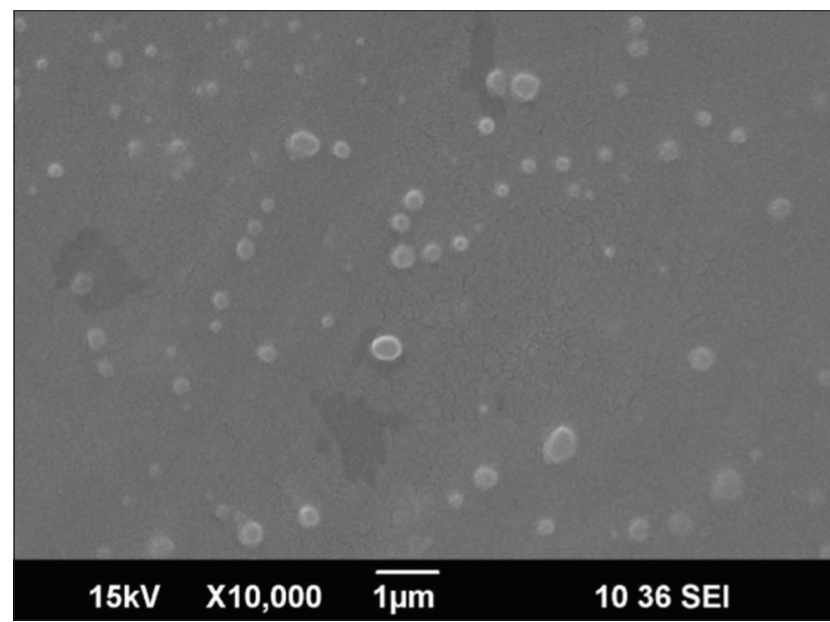

Fig. 5: Scanning electron microscopy of optimized formulation

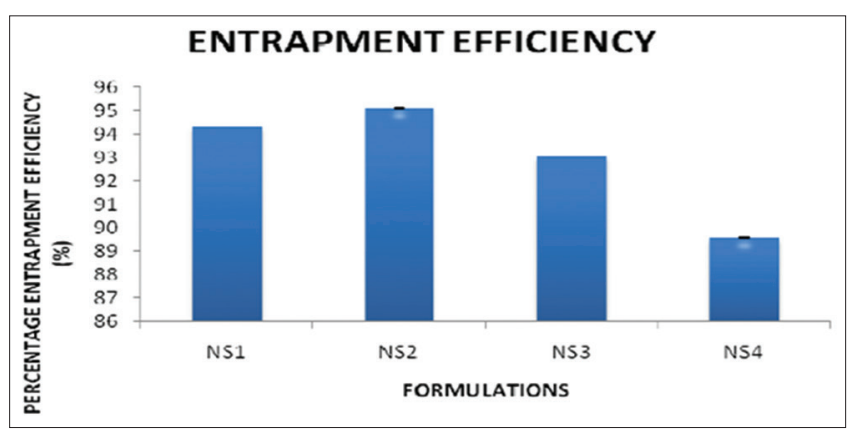

Fig. 6: Representing entrapment efficiency of formulations in the entrapment efficiency while kept for 45 days in both of these conditions represented in Fig. 14.

\section{CONCLUSION}

The current article was intended to build up an appropriate transporter for the delivery of cefixime, third-generation cephalosporin antibiotic drug to conquer the troubles associated with it, like the poor solubility, low bioavailability, and half-life of the drug, thereby increasing the duration of action and thereby increasing the half-life. The preformulation studies were carried out; from the study, it was clear that it satisfy the entire characteristic for oral drug delivery. The partition coefficient of the drug confirmed that the drug is lipid soluble and hence confirmed that niosomal suspension may be a proficient for the delivery of drug. The niosomal suspension was prepared by thin film hydration technique by varying the concentration of surfactant, while keeping the concentration of cholesterol same. The characterization of the niosomal suspension was conducted and had a particle size of 350-450 nm. In vitro drug release was done in both stomach $\mathrm{pH}$ and intestinal $\mathrm{pH}$. The release in stomach $\mathrm{pH}$ was only $0.4 \%$, while the release in intestinal $\mathrm{pH}$ was $57.72 \%$. The effect of gastrointestinal tract enzymes such as pepsin and trypsin on the in vitro liberation of drug from the formulation was also carried out. It was found that there is no much significant effect of the enzymes on the release of the drug from the formulation. The kinetics study of the formulation follows Higuchi model which states that the distance of the diffusion increases the release of drug will be at a slower pace. This concludes that the drug is being released from the inner side of niosomal suspension in a controlled manner. The optimization was based on the entrapment efficiency and in vitro release studies. ANOVA showed that NS2 (Span 60-300 $\mathrm{mg}$ and cholesterol - $200 \mathrm{mg}$ ) is the optimized formulation. In vitro antibacterial study against $E$. coli and Klebsiella revealed that the zone of inhibition higher than the marketed formulation. The MIC confirmed that the inhibitory concentration was much less $0.05 \mathrm{mg}$; hence, it can be used as a proficient transporter for the delivery of cefixime. The formulation was stable for about 45 days at two different temperatures. From the present study, it is clear that the surfactant at the medium level showed highest entrapment efficiency and release. The in vitro antibacterial activity showed that the activity is more than the marketed formulation and the MIC was much lower than the

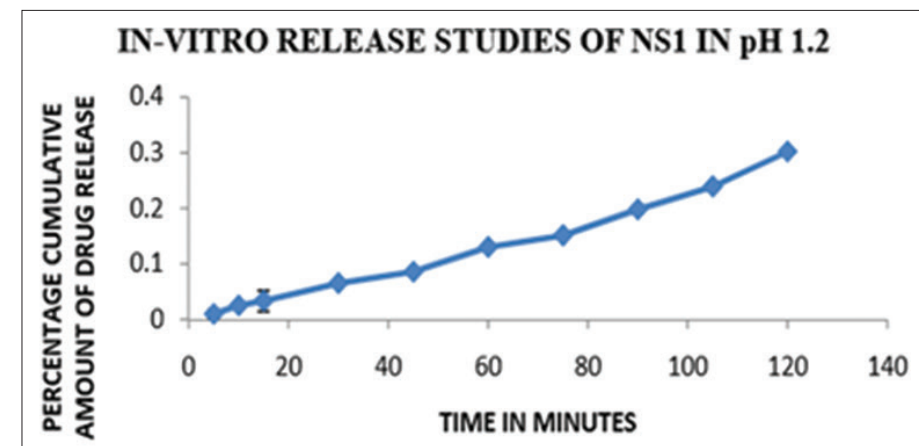

\section{IN-VITRO RELEASE STUDIES OF NS3 IN pH 1.2}

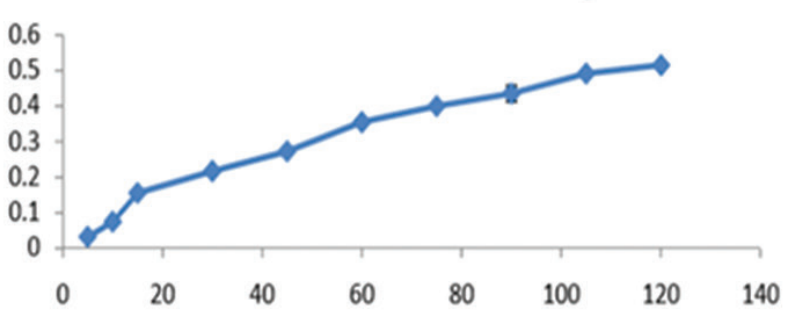

TIME IN MINUTES

\section{IN-VITRO RELEASE STUDIES OF NS2 IN pH 1.2}

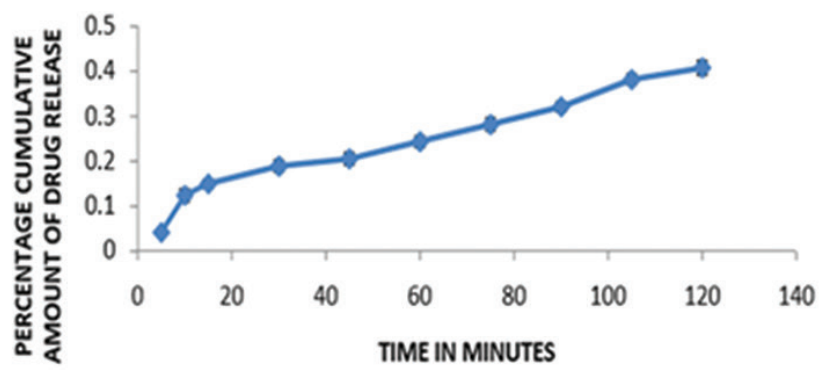

IN-VITRO RELEASE STUDIES OF NS4 IN pH 1.2

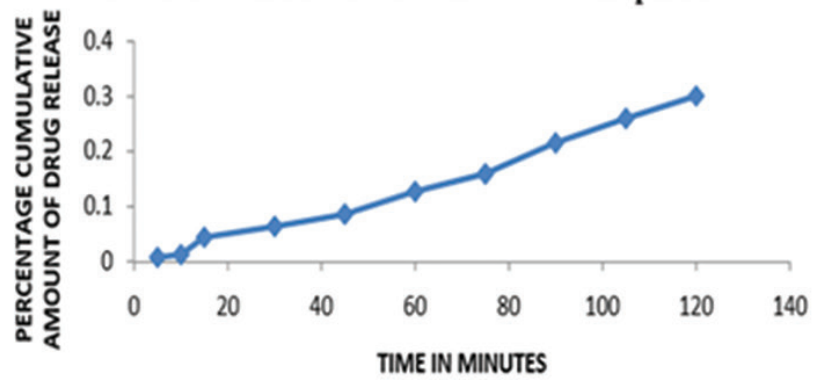

Fig. 7: In vitro release studies of NS1, NS2, NS3, and NS4 in pH 1.2 


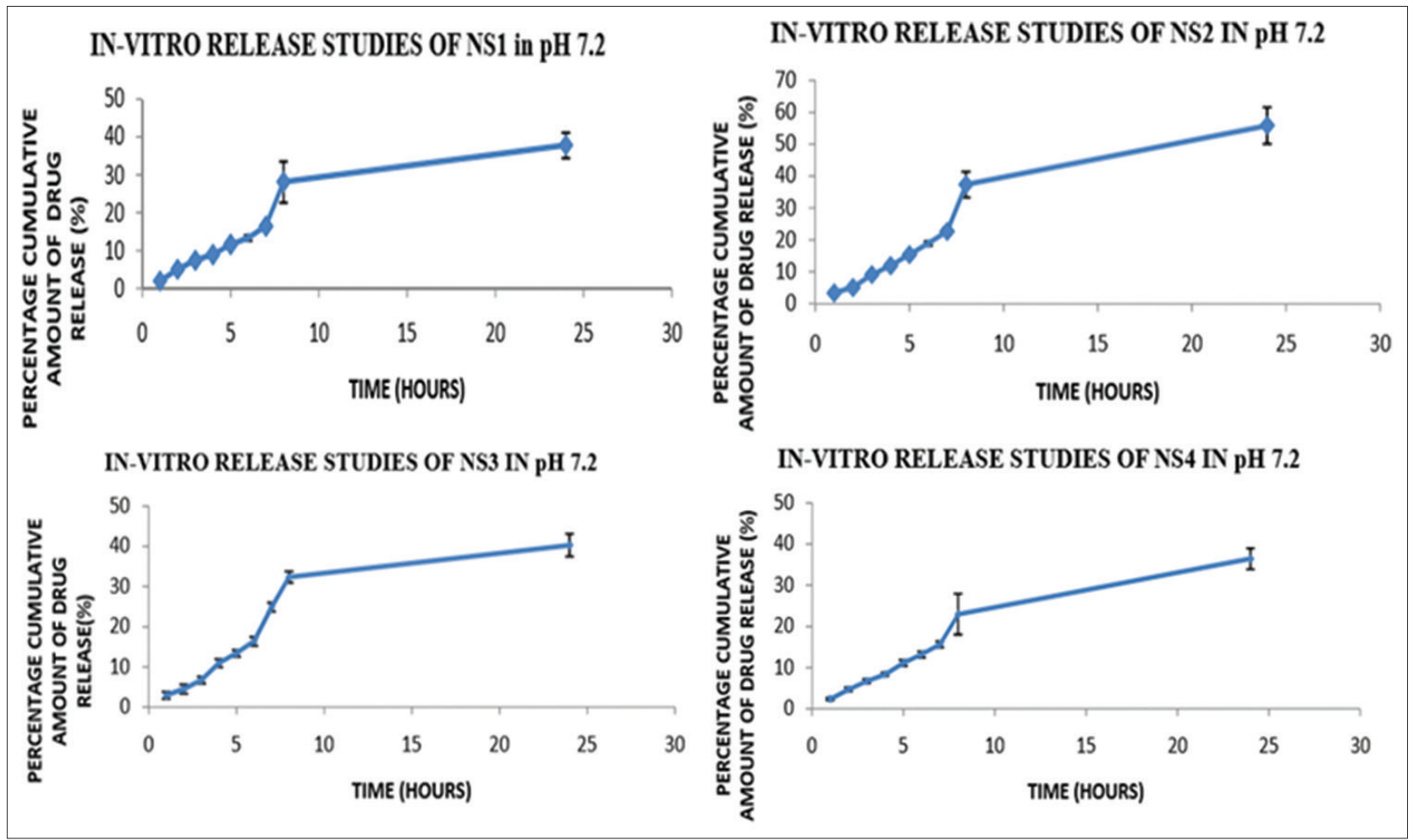

Fig. 8: In vitro release studies of NS1, NS2, NS3, and NS4 in pH 7.2

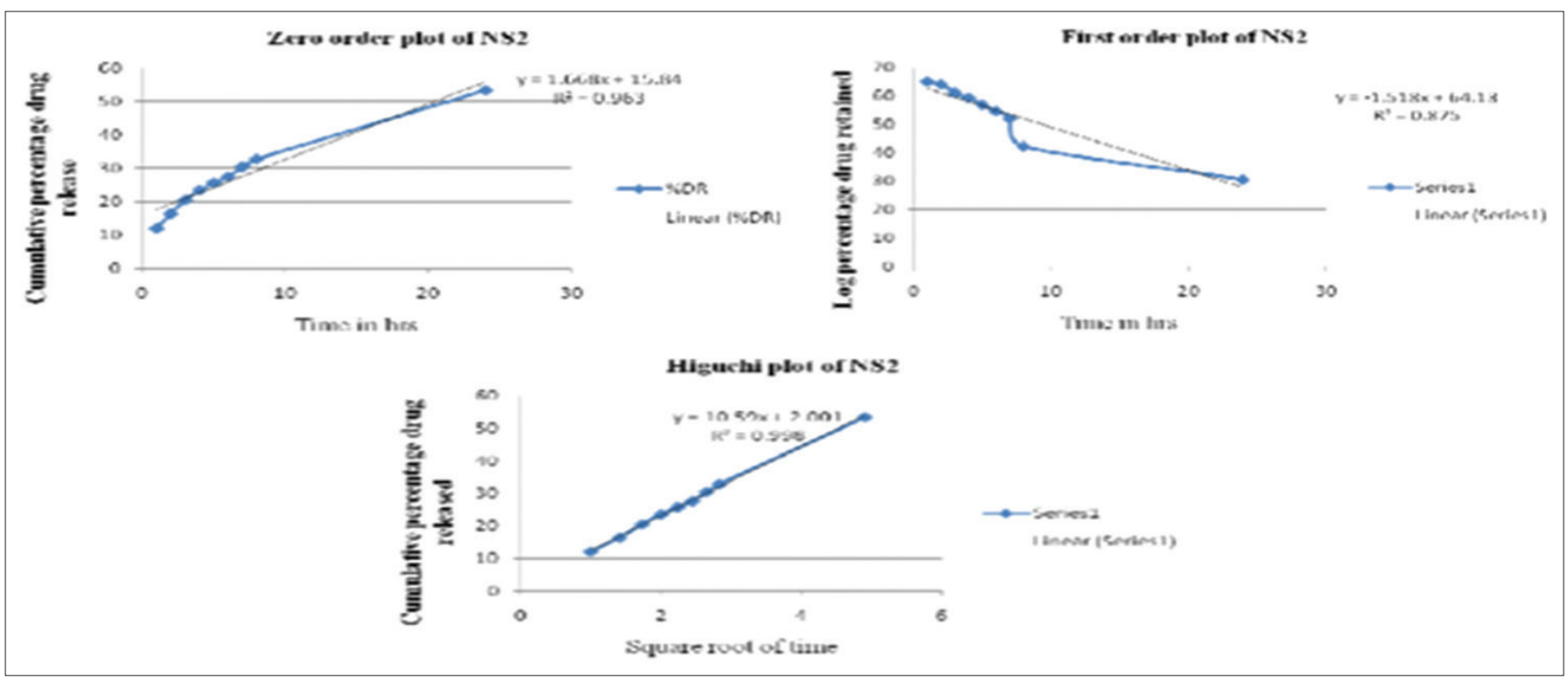

Fig. 9: Zero order, first order, and Higuchi plot of NS2

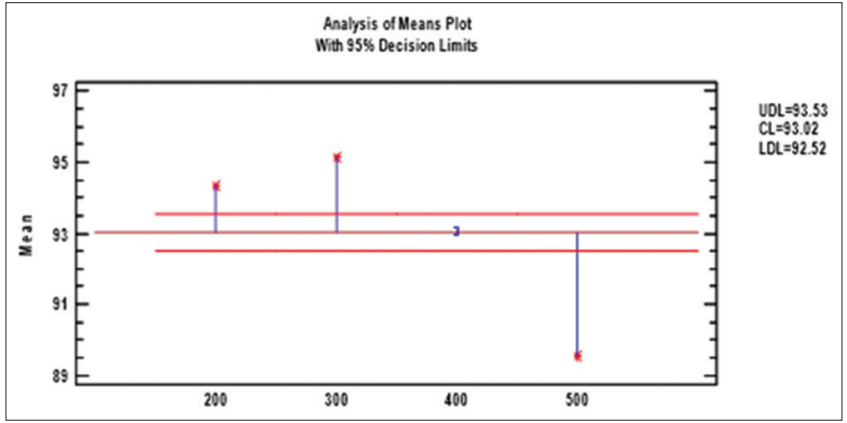

Fig. 10: ANOVA analysis of entrapment efficiency
Table 1: Composition of formulation

\begin{tabular}{lllll}
\hline \multirow{2}{*}{ Ingredients } & \multicolumn{3}{l}{ Formulation code } & \\
\cline { 2 - 5 } & NS1 & NS2 & NS3 & NS4 \\
\hline Surfactant (mg) & 200 & 300 & 400 & 500 \\
Cholesterol (mg) & 200 & 200 & 200 & 200 \\
Chloroform (ml) & 20 & 20 & 20 & 20 \\
Phosphate buffer (7.2) (ml) & 20 & 20 & 20 & 20 \\
Drug (mg) & 20 & 20 & 20 & 20 \\
\hline
\end{tabular}

standard used. Thus, niosomal suspension is a promising carrier for the delivery of cefixime. 
Table 2: Evaluation of in vitro antibacterial activity by zone of inhibition

\begin{tabular}{|c|c|c|c|c|}
\hline \multirow[t]{2}{*}{ Microorganism } & \multirow[t]{2}{*}{ Concentration ( $\mu \mathrm{l} /$ well) } & \multicolumn{3}{|l|}{ Zone of inhibition (mm) } \\
\hline & & Marketed formulation & Optimized formulation & Plain niosomes \\
\hline \multirow[t]{3}{*}{ E. coil } & 2 & 0 & 0 & 0 \\
\hline & 10 & 14 & 18 & 0 \\
\hline & 15 & 16 & 2 & 0 \\
\hline \multirow[t]{4}{*}{ Klebsiella } & 2 & 0 & 0 & 0 \\
\hline & 5 & 1 & 14 & 0 \\
\hline & 10 & 12 & 16 & 0 \\
\hline & 15 & 14 & 19 & 0 \\
\hline
\end{tabular}

E. coli: Escherichia coli

Table 3: Minimum inhibitory concentration of cefixime loaded niosomal suspension

\begin{tabular}{ll}
\hline Microorganisms & Minimum inhibitory concentration $(\mathrm{mg} / \mathrm{ml})$ \\
\cline { 2 - 2 } & Optimized formulation \\
\hline E. coli & $0.05 \mathrm{mg}$ \\
Klebsiella & $0.05 \mathrm{mg}$ \\
\hline
\end{tabular}

E. coli: Escherichia coli

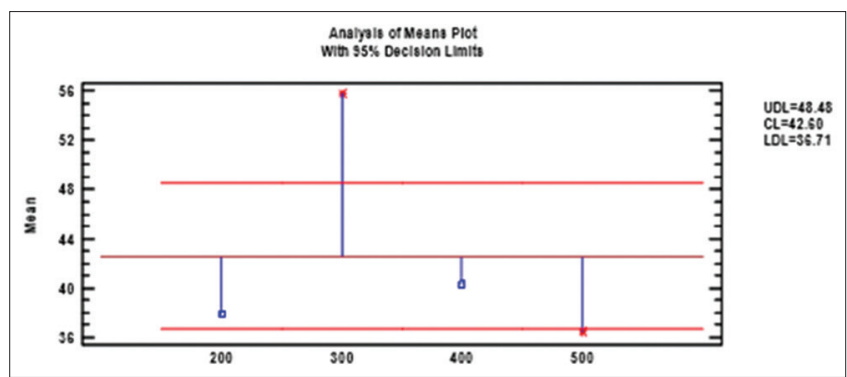

Fig. 11: ANOVA analysis of release study in vitro

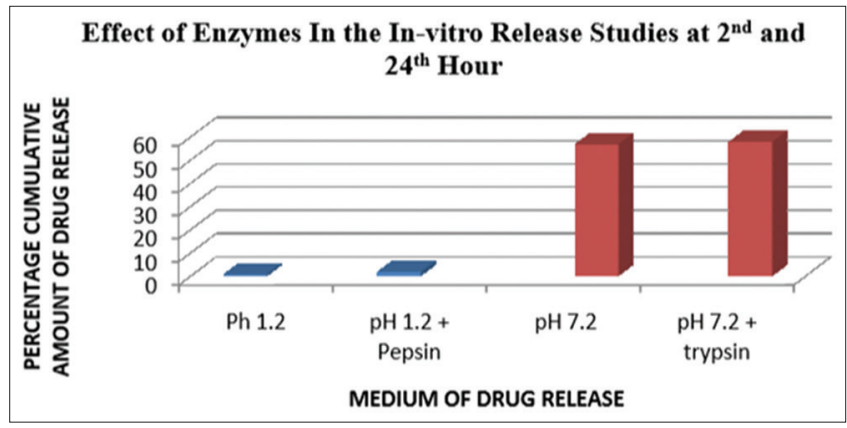

Fig. 12: Effect of enzymes in the in vitro release of optimized formulation

\section{REFERENCE}

1. Rajnish A, Ajay S. Release studies of ketoprofen niosome formulation. J Chem Pharm Res 2010;2;79-82.

2. Sandeep KS, Meenakshi C, Anilkumar N. Span-60 niosomal oral suspension of fluconazole: Formulation and in vitro evaluation. J Pharm Res Health Care 2009;1:142-56.

3. Pranshu $T$, Shaffi K. Niosomes: Formulation and evaluation. Int $\mathrm{J}$ Biopharm 2011;2:47-53.

4. Rogerson A, Cummings J, Willmott N, Florence AT. The distribution of doxorubicin in mice following administration in niosomes. J Pharm Pharm 1998;40:337-42.

5. Baillie AJ, Coombs GH, Dolan TF. Non-ionic surfactant vesicles, niosomes as delivery system for the anti-leishmanial drug sodium stribogluconate. J Pharm Pharm 1986;38:502-5.

6. Chauhan S, Luorence MJ. The Preparation of polyoxyethylene

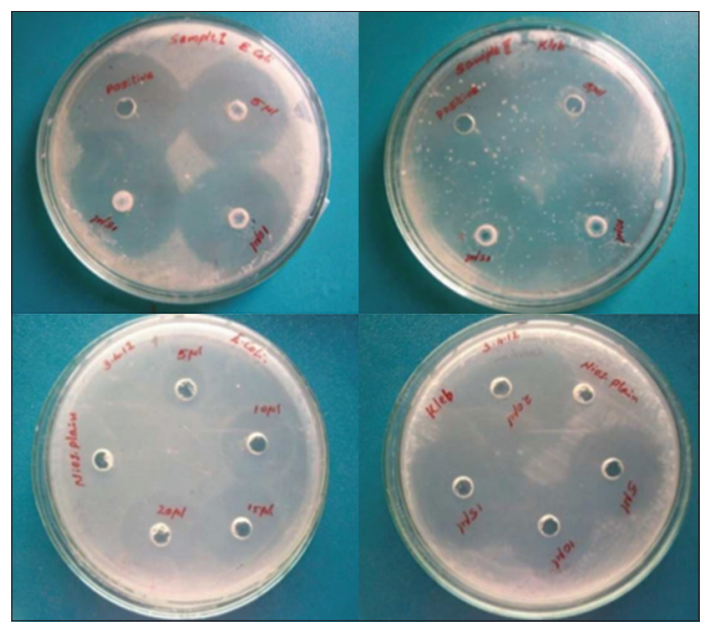

Fig. 13: Photograph of in vitro antibacterial activity of optimized formulation and photograph of in vitro antibacterial activity of optimized formulation

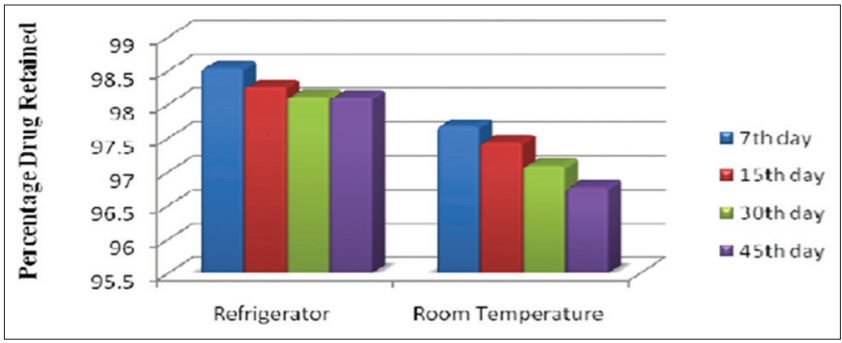

Fig. 14: Stability study of optimized formulation NS2

containing non-ionic surfactant vesicles. J Pharm Pharm 1989;41:6-10.

7. Blazek-Walsh AI, Rhodes DG. SEM imaging predicts quality of niosomes from maltodextrin-based proniosomes. Pharm Res 2001;18(23):656-61.

8. Nair AS, Vidhya KM, Saranya TR, Sreelakshy KR, Nair SC. Emulsomes: A novel liposomal formulation for sustained drug delivery. Int Res J Pharm Appl Sci 2013;3(5);192-6.

9. Naresh RA, Chandrashekhar G, Pillai GK, Udupa N. Antiinflammatory activity of niosome encapsulated diclofenac sodium with tween- 85 in arthitic rats. Indian J Pharm 1994;26:46-8.

10. Khandare JN, Madhavi G, Tamhankar BM. Niosomesnovel drug delivery system. Eastern Pharm 1994;37:61-4.

11. Hunter CA, Dolan TF, Coombs GH, Baillie AJ. Vesicular systems (Niosomes and liposomes) for delivery of sodium stibogluconate in experimental murine visceral leishmaniasis. J Pharm Pharm 1988;40:161-5.

12. Malhotra M, Jain NK. Niosomes as drug carriers. Indian Drugs 1994;31:81-6

13. Baby AA, Saroja S, Sabitha M. Mechanism of solubility of liquisolid formulation in non-volatile solvent: A review. Int J Pharm Pharm Sci 2012;4:710-5

14. Raghavendra NR, Ram P, Bussetti SS. Formulation and evaluation of 
gas powered systems of cefixime tablets for controlled release. Int $\mathbf{J}$ Pharm Bio Sci 2010;1:12-6.

15. Shivanand P. Development and characterization of cefpodoxime proxetil niosomes. Int J Pharm World Res 2010;1:12-23.

16. Barbara L, Federica B, Teresa C, Roberto M, Anna, MD, Vittorio Z. New environmental sensitive system for colon-specific delivery of peptidic drugs. Int J Pharm 2008;358:44-9.

17. Shivakumar HN, Siva RK, Narasimha SM. Albumin microspheres for oral delivery of iron. J Drug Target 2010;18:36-44.
18. Solmaz G, Jaleh V, Afrooz S, Fatemeh A. Stability and antimicrobial effect of amikacin-loaded solid lipid nanoparticles. Int J Nanomed 2011;6:35-43.

19. Nair SC, Anoop KR. Local antimicrobial delivery of satranidazole loaded cross linked periodontal chips using bio degradable polymers. Int J Pharm Pharm Sci 2013;5(3):839-47.

20. Nair AS, Vidhya KM, Saranya TR, Sreelakshmy KR, Nair SC Mucoadhesive buccal patch of cefixime trihydrate using biodegradable natural polymer. Int J Pharm Pharm Sci 2014;6(6):366-71. 